\title{
Glucose not good for the gut
}

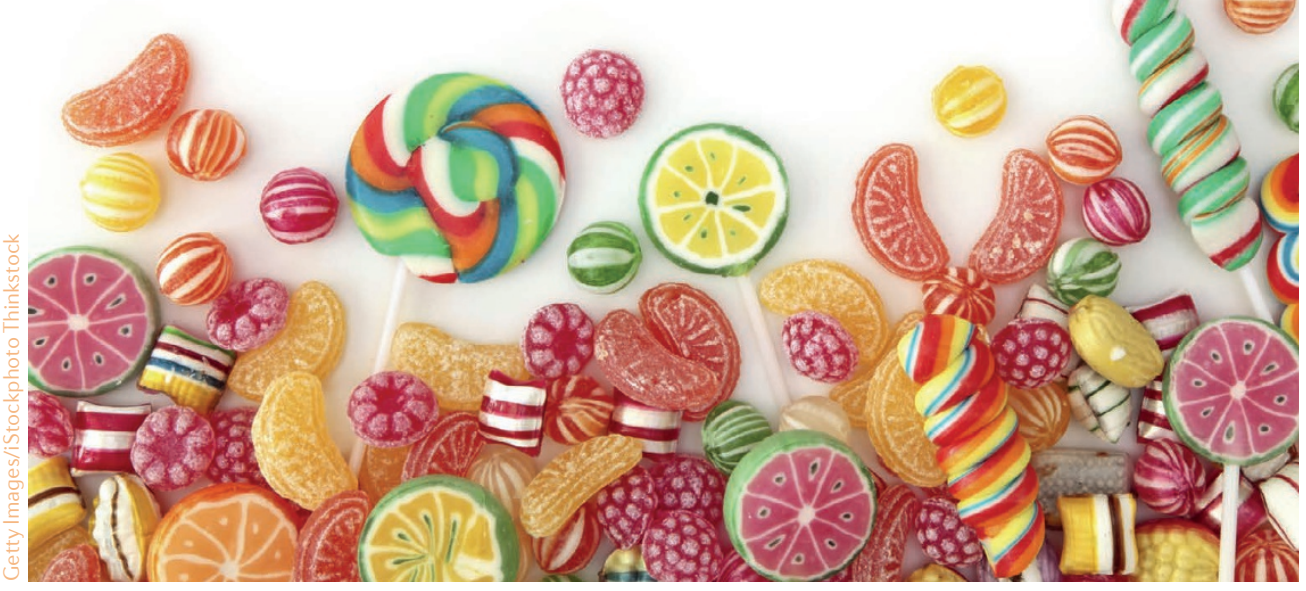

Obesity and the associated metabolic syndrome have been linked to chronic inflammatory processes in various tissues, as well as an increased general risk of infection. This state of 'metaflammation' is thought to result from intestinal barrier dysfunction, leading to the translocation of microbial products into the systemic circulation. Eran Elinav and colleagues show that hyperglycaemia, rather than obesity itself, provides the mechanistic basis for this barrier dysfunction.

In mice with genetic dysfunction of the leptin receptor ( $d b / d b$ mice) or leptin deficiency ( $o b / o b$ mice), which result in over-eating and obesity, increased levels of microbial ligands were detected at multiple systemic sites. RNA sequencing of colonic tissue showed that these mice had global changes in transcription, including markedly reduced expression of genes involved in junctional integrity that would normally inhibit the para-cellular influx of intestinal molecules into the lamina propria. As a result, $d b / d b$ mice and $o b / o b$ mice were susceptible to systemic colonization with Citrobacter rodentium, which causes a self-limiting infection restricted to the gut in wild-type mice.

Bone marrow chimaera experiments showed that nonhaematopoietic cells are responsible for mediating resistance to C. rodentium colonization. Leptin receptor expression has been reported on cells of the gut, liver and nervous systems, but mice with specific deletion of leptin receptor in these cell types did not consistently have increased susceptibility to $C$. rodentium, which suggests that the leptin signalling pathway cannot by itself account for barrier function. Similarly, obesity was ruled out as a direct driver of barrier dysfunction. When food access for $d b / d b$ mice was restricted to the amount consumed by wild-type mice, they were still susceptible to systemic $C$. rodentium infection.

Another common feature of metabolic syndrome is glucose intolerance and hyperglycaemia. Indeed, all of the mouse models that were susceptible to $C$. rodentium had increased blood glucose levels. To test the effects of hyperglycaemia in the absence of obesity, the authors used a mouse model of streptozotocin (STZ)-induced type 1 diabetes. STZ-treated mice had decreased junctional integrity between intestinal epithelial cells (IECs), increased translocation of microbial products and increased susceptibility to $C$. rodentium. When STZ-treated mice were administered insulin to restore normoglycaemia, barrier integrity and resistance to C. rodentium were also restored. Thus, hyperglycaemia is a specific and direct cause of intestinal barrier dysfunction.

Compared with controls, IECs from STZ-treated mice had differential expression of genes involved in metabolic pathways, particularly those that are known to be involved in the maintenance of epithelial barrier function, such as N-glycan biosynthesis. This transcriptional reprogramming in STZ-treated mice was blocked by the inhibition of glucose metabolism using 2-deoxyglucose, which restored barrier function and host defence against $C$. rodentium. Mice lacking the bidirectional glucose transporter GLUT2 in IECs were resistant to STZ-induced changes.

In summary, the mouse studies suggest that hyperglycaemia leads to GLUT2-mediated glucose transport into IECs and their transcriptional reprogramming, which decreases barrier integrity and accounts for systemic infection and inflammation through the increased translocation of bacteria and their products. In 27 healthy humans, an individual's average plasma glucose concentration had the strongest correlation - compared with other factors such as body mass index - with serum levels of microbial ligands. It will be interesting to determine if shorterterm effects of a high-glucose diet may also alter intestinal barrier function.

Kirsty Minton

ORIGINAL ARTICLE Thaiss, C. A. et al. Hyperglycemia drives intestinal barrier dysfunction and risk for enteric infection. Science https://doi.org/10.1126/science.aar3318 (2018) 